\title{
Problem solving with adaptive feedback
}

\author{
Rainer Lütticke \\ FernUniversität Hagen, Department of Computer Science, \\ Intelligent Information and Communication Systems, 58084 Hagen, Germany, \\ Rainer.Luetticke@fernuni-hagen.de, \\ WWW home page: http://pi7.fernuni-hagen.de/luetticke/
}

\begin{abstract}
The virtual laboratory (VILAB) supports interactive problem solving in computer science with access to complex software-tools. During the problem solving processes the learners get fast feedback by a tutoring component. This feedback based in the first version of VILAB only on intelligent error analyses of learners' solutions. Animated by the very positive results of the evaluation of this tutoring component we additionally implemented an user model. Thereby the feedback for a learner consists not only of adaptive information about his errors and performance, but also of adaptive hints for the improvement of his solution. Furthermore, the tutoring component can individually motivate the learners.
\end{abstract}

\section{Introduction}

We have developed an Internet-based virtual laboratory $\left(\right.$ VILAB $\left.^{1}\right)$ for computer science instructing practical courses in online education at the largest German open and distance university (FernUniversität Hagen) [4]. Since summer 2002 this learning environment is in use for regular teaching.

VILAB is realised in a client-server architecture in which the Linux-based server is operated at the FernUniversität. The access to the server is managed by decentral user clients which must be connected with the Internet. Via remotelogin to the server the users are directly routed to a navigation tool which is one of two user interfaces. The second user interface is a common browser opened on a client with an individual URL for every user. Via navigation tool local hypertext (e.g. problems) as well as documents out of the WWW can be selected and displayed in the browser. Complex graphical software-tools (e.g. SNNS ${ }^{2}$ or $\mathrm{MWR}^{3}$ ) installed on the server can also be activated via navigation tool.

While the most adaptive learning environments are focused on the optimal presentation and sequencing of content VILAB is focused on problem solving like Andes, an intelligent tutoring system for physics [3]. The problems in VILAB are divided in different domains of computer science (e.g. relational data bases, neural networks, automatical natural language processing). The solutions are

\footnotetext{
${ }^{1}$ http://pi7.fernuni-hagen.de/vilab/

${ }^{2}$ Stuttgart Neural Network Simulator

3 tool for knowledge representation of multilayered extended semantic networks [4]
} 
automatically analysed by the system and a tutoring component gives feedback for immediate improvements [3,6]. After this the learner modifies his solution and a further analysis starts. This interaction lasts until the solution is correct. ${ }^{4}$

\section{Analyses of solutions and the resulting feedback}

There exist different modes for the creation of the solution of a problem. For the standard tests (e.g. multiple choice test, asking for numbers, correlations, etc.) we use the WebAssign-System ${ }^{5}$ [4]. Text areas in hypertext documents or in software-tools are used for free text (e.g. SQL queries or reformulation of semantic networks respectively). The most complex input mode of a solution is a graphic (e.g. building of neural or semantic networks) which can be created with the software-tools.

The tutoring component has a passive and an active error mode for two different kinds of problem solving support [1]. In the active mode ("interactive problem solving support") the tutoring component does not wait for the learner's final solution. If an error is interactively detected (e.g. action in a software-tool or "click" in a hypertext document leading to a wrong solution), it immediately sends information about the errors either in a newly displayed window or by graphical reactions. These reactions of the tutoring component are short, often technical, and hardly possess variants.

In the passive mode ("intelligent analysis of student solutions") the tutoring component has to identify errors from the learner's final result after he has requested for a correction or support [6]. The analysis of the learner's solution is realised by correction modules using different methods. Depending on the problem they can test the learner's solution and confront him with the consequences of his errors [6] (e.g. test of results of SQL-queries) or compare the solution with sample solution(s) by term or pattern analysis (similar to [5], but not so elaborated) or inferences. Additionally, the modules use a table with deductions from certain errors and a table with hints for understanding certain concepts and for prevention of certain errors based on experiences of teachers. Thereby the feedback of an analysis is adaptive to the individual errors.

The elements of the feedback (s. references in [4]) consist of a statement about the degree of correctness of the solution, an error list, a description and explanation of errors, hints for improvement of the solution and the avoidance of errors, a listing of useful literature or lectures to derive lacking knowledge, a link to an easier problem which is connected with the actual problem, examples of similar exercises, an assessment of the learner's performance, and a motivation.

The output of an analysis is an error code with accompanying parameters. The content of these parameters is not a fixed number of possibilities, but based on the content of the learner's solution and its intelligent analysis. The error code is connected with a hypertext template including Javascript variables filled out by the parameters. The final hypertext document is copied to the individual

\footnotetext{
${ }^{4}$ Guided Tour: http://ki219.fernuni-hagen.de/tour_en/

${ }^{5}$ http://niobe.fernuni-hagen.de/WebAssign/
} 
URL of the user. This result in an automatical reload in the browser of the user managed by PHP scripts and the display of the feedback [4].

\section{$3 \quad$ User model}

In winter term 2003/04 we added to the system an user model based on information collected in a data base. The elements of the model are: 1. learning type, 2. knowledge level, 3. solved problems in VILAB, 4. number of attempts to solve a problem, 5. distance of a learner's solution to a correct solution, and 6. the changing of this distance between two attempts. The last two elements are used for the monitoring of the learner while he is solving a problem [7]. The user model can combine information about the current state of the problem solving process (elements 4, 5, and 6) with long-term assessment of the learner's knowledge of computer science (elements 2 and 3) similar to the system Andes [3].

The learning type is determined by a multiple choice test before the learner begins to work in VILAB. The types (text oriented, examples oriented, and interaction oriented) are defined by the demands of learners on an e-learning system [2]. The knowledge level is determined by previously successful courses and result in locking problems and domains of VILAB. The distance is computed from the learner's solution to the sample solution(s) by individual measurements for every problem (e.g. number of wrong nodes and relations between nodes in semantic networks).

\section{Adaptive features based on the user model}

Due to the "knowledge level" of a learner the system can automatically unlock problems of VILAB depending on the individual progress of problem solving. In this way, every learner gets access to problems adaptively fitting to his experience and practical background knowledge.

Including PHP code in the hypertext templates and filling PHP variables by data from the user model the feedback becomes adaptive for the learner. Depending on the values of the user model the parameters of the analysis of the learner's solution are displayed or masked, or they are modified. The input for these "display-functions" are the values of the elements of the user model. Three constraints are exemplary listed. A constraint for the display-functions is the dependency between learning type and the instruction method to improve solutions. Another constraint exists between the number of unsuccessful attempts to solve a problem, the distance of a solution to the correct solution, and the changing of this distance between two attempts on the one side and the extent and description of the error list on the other side. The content of the motivation is determined by nearly all values of the user model.

Using the models in this way the feedback is adaptive to the individual errors as well to the user's knowledge, preferences in support, and progress in solving the problem. 


\section{$5 \quad$ Evaluation and perspective}

Results of evaluations of VILAB without user models (40 questionnaires and informal interviews) show that the students like the kind of learning with the interactive tutoring component because the fast feedback gives the possibility to learn in "trial and error"-mode which $\sim 90 \%$ have used. The support of the tutoring component during the finding of the correct solution is important for $\sim 80 \%$ of the students. Although the component was helpful $\sim 40 \%$ wish a more adaptive feedback. Its motivation to find the right solution is helpful to prevent to give up the search for the right solution $(\sim 30 \%)$. Comparing courses using VILAB and similar courses in former times without this learning environment the rate of successful finishers increases to $\sim 50 \%$. Additionally, from the view of the teacher learning with VILAB is connected with better results in examinations and better seminar talks. The tutoring component is so effective that newsgroups or emails to the teacher in courses with VILAB were $\sim 50 \%$ less used.

With the implementation of the user model in VILAB and with its use in courses beginning in summer 2004 we think that we can further improve the learning success of the students. The more adaptive feedback will strengthen the positive effects of the tutoring component.

\section{References}

1. Brusilovsky, P.: Adaptive and Intelligent Technologies for Web-based Education. Künstliche Intelligenz 13(4) (1999) 19-25

2. Ehlers, U.-D.: Quality in e-learning from a learner's perspective. In: Benrath, U. and Szücs, A. (eds.), Supporting the Learner in Distance Education and E-Learning, Proceedings of the 3rd EDEN Research Workshop, Bibliotheks- und Informationssystem der Universität Oldenburg (BIS) (2004) 130-137

3. Gertner, A.S., VanLehn, K.: Andes: A Coached Problem Solving Environment for Physics. In: Gauthier G., Frasson C., and VanLehn K. (eds.), Intelligent Tutoring Systems 2000, LNCS 1839, Springer (2000) 133-142

4. Lütticke, R., Helbig, H.: Practical courses in distance education supported by an interactive tutoring component. In: Benrath, U. and Szücs, A. (eds.), Supporting the Learner in Distance Education and E-Learning, Proceedings of the 3rd EDEN Research Workshop, Bibliotheks- und Informationssystem der Universität Oldenburg (BIS) (2004) 441-447

5. Martin, B., Mitrovic, A.: Tailoring Feedback by Correcting Student Answers. In: Gauthier G., Frasson C., and VanLehn K. (eds.), Intelligent Tutoring Systems 2000, LNCS 1839, Springer (2000) 383-392

6. Mathan, S., Koedinger K.R.: Recasting the Feedback Debate: Benefits of Tutoring Error Detection and Correction Skills. In: Hoppe, U., Verdejo, F., and Kay, J. (eds.), Artificial Intelligence in Education, Frontiers in Artificial Intelligence and Applications Vol. 97, IOS Press (2003) 13-20

7. Verdejo, M.F.: A Framework for Instructional Planning and Discourse Modeling in Intelligent Tutoring Systems. In: Costa, E. (ed.), New Directions for Intelligent Tutoring Systems, Springer (1992) 147-170 